\title{
Dissipation and velocity distribution at the shear-driven jamming transition
}

\author{
Peter Olsson \\ Department of Physics, Umeå University, 90187 Umeå, Sweden
}

(Dated: October 16, 2018)

\begin{abstract}
We investigate energy dissipation and the distribution of particle velocities at the jamming transition for overdamped shear-driven frictionless disks in two dimensions at zero temperature. We find that the dissipation is caused by the fastest particles and that the fraction of particles responsible for the dissipation decreases towards zero as jamming is approached. These particles belong to an algebraic tail of the velocity distribution that approaches $\sim v^{-3}$ as jamming is approached. We further find that different measures of the velocity diverge differently, which means that concepts like "typical velocity" may no longer be used - a finding that should have implications for analytical approaches to shear-driven jamming.

PACS numbers: 64.60.-i, 64.70.Q-, 45.70.-n
\end{abstract}

The hypothesis that the slowing down of the dynamics in systems as different as supercooled liquids, granular materials, colloids, foams, and emulsions, have a common origin in the properties of a critical point, point $\mathrm{J}[1]$, has inspired a great amount of work on jamming the last decade. Several models have been used to try and pinpoint the properties of this jamming transition. Some of them have centered around a greatly simplified numerical model of spheres with contact-only interaction. One important branch has been to examine the properties of randomly generated static packings[2] whereas another has been to study the jamming transition through simulations of elastic particles under steady shear[3].

A key feature of jamming is the approach of the contact number $z$ to the isostatic number $z_{\text {iso }}$ which is just enough for mechanical stability. It has recently been shown[4] that this is directly linked to the divergence of $\eta_{p} \equiv p / \dot{\gamma}$ - the pressure equivalent of the shear viscosity. A related phenomenon is the increase in particle velocity as $\phi \rightarrow \phi_{J}[5,6]$. This is related to the distribution of particle displacements due to a small shear increment which has been determined both in experiments of sheared granular materials $[7]$ and in quasistatic simulations $[5,7]$. It was there found that this distribution is sufficiently wide that the non-Gaussian parameter $\left\langle\Delta y^{4}\right\rangle / 3\left\langle(\Delta y)^{2}\right\rangle-1$ diverges as $\phi_{J}$ is approached from below, granted that the shear step is sufficiently small.

In this Letter we show that there is more to the particle velocity distribution than has so far been realized. Dissipation is mainly caused by the fastest particles and we find that the fraction of particles that are responsible for the dissipation decreases towards zero as jamming is approached. This behavior is related to an algebraic tail, $P(v) \sim v^{-3}$, in the velocity distribution and we show that the velocity histograms determined at the jamming density approach this limiting behavior as $\dot{\gamma} \rightarrow 0$. Since rheology and dissipation are linked through power balance, the understanding of this phenomenon is right at the center of the phenomenon of shear-driven jamming. We also note that this finding has profound consequence for ana- lytical approaches to jamming since it implies that different measures of the velocity behave differently and that concepts like "typical velocity" therefore become useless.

Following O'Hern et al.[2] we use a simple model of bi-disperse frictionless soft disks in two dimensions with equal numbers of disks with two different radii in the ratio 1.4. Length is measured in units of the diameter of the small particles, $d_{s}$. We use Lees-Edwards boundary conditions[8] to introduce a time-dependent shear strain $\gamma=t \dot{\gamma}$. With periodic boundary conditions on the coordinates $x_{i}$ and $y_{i}$ in an $L \times L$ system, the position of particle $i$ in a box with strain $\gamma$ is defined as $\mathbf{r}_{i}=\left(x_{i}+\gamma y_{i}, y_{i}\right)$. The ordinary velocity is $\mathbf{v}_{i}^{\text {tot }}=\dot{\mathbf{r}}_{i}$, but in the following we consider the non-affine velocity, $\mathbf{v}_{i}=\mathbf{v}_{i}^{\text {tot }}-\mathbf{v}_{\mathrm{R}}\left(\mathbf{r}_{i}\right)$ where $\mathbf{v}_{\mathrm{R}}\left(\mathbf{r}_{i}\right) \equiv \dot{\gamma} y_{i} \hat{x}$ is a uniform shear velocity. With $r_{i j}$ the distance between the centers of two particles and $d_{i j}$ the sum of their radii, the relative overlap is $\delta_{i j}=1-r_{i j} / d_{i j}$ and the interaction between overlapping particles is $V\left(r_{i j}\right)=\epsilon \delta_{i j}^{2} / 2$; we take $\epsilon=1$. The force on particle $i$ from particle $j$ is $\mathbf{f}_{i j}^{\text {el }}=-\nabla_{i} V\left(r_{i j}\right)$. The simulations are performed at zero temperature.

We consider two different models for the energy dissipation. In both cases the interaction force is $\mathbf{f}_{i}^{\mathrm{el}}=\sum_{j} \mathbf{f}_{i j}^{\mathrm{el}}$ where the sum extends over all particles $j$ in contact with $i$, and the equation of motion is

$$
\mathbf{f}_{i}^{\mathrm{el}}+\mathbf{f}_{i}^{\mathrm{dis}}=m_{i} \ddot{\mathbf{r}}_{i} .
$$

Most of our simulations have been done with the $\mathrm{RD}_{0}$ (reservoir dissipation) model with the dissipating force

$$
\mathbf{f}_{\mathrm{RD}, i}^{\mathrm{dis}}=-k_{d} \mathbf{v}_{i} .
$$

We take $k_{d}=1, m_{i}=0$, and the time unit $\tau_{0}=d_{s} k_{d} / \epsilon$. We simulate $N=65536$ particles with shear rates down to $\dot{\gamma}=10^{-10}$. Checking for finite size effects at $\dot{\gamma}=10^{-9}$ we found no difference when using instead $N=262144$. The equations of motion were integrated with the Heuns method with time step $\Delta t=0.2 \tau_{0}$.

Some additional simulations have also been done with the $\mathrm{CD}_{0}$ model ( $\mathrm{CD}$ for "contact dissipation") with dissi- 

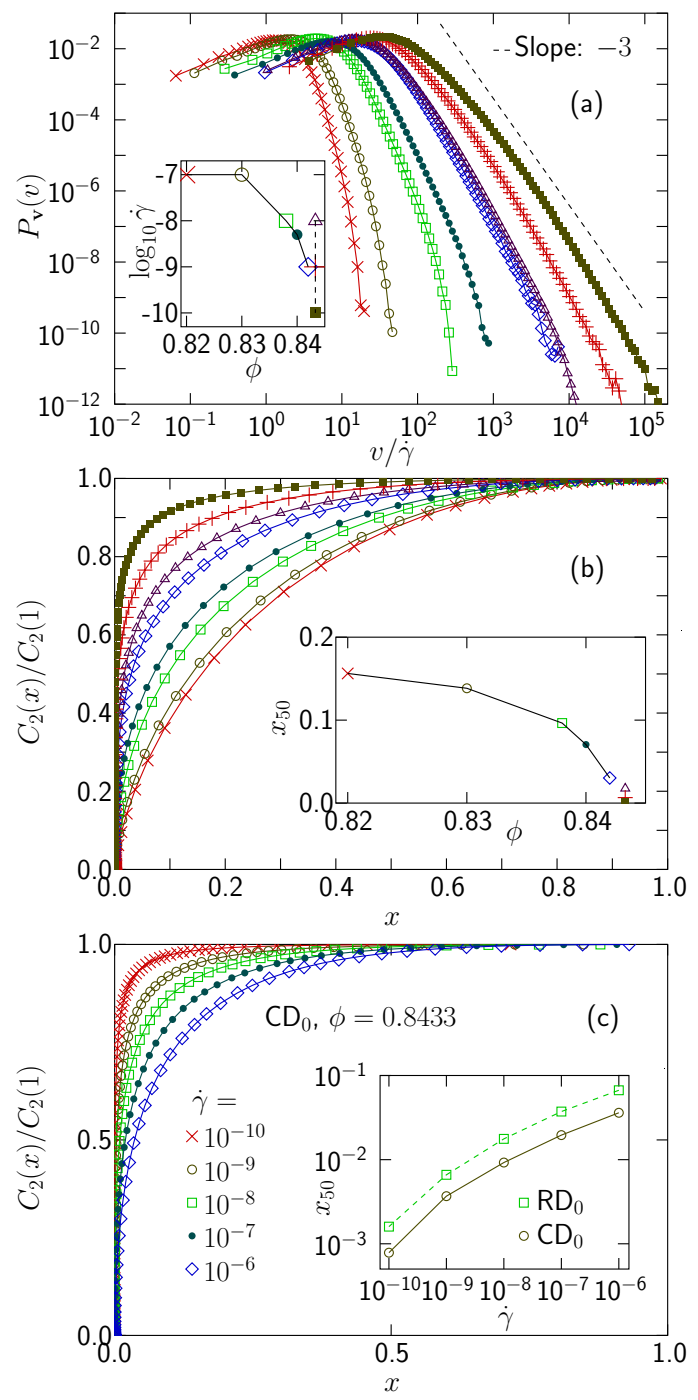

FIG. 1. (Color online) Velocity distribution and dissipation. Panel (a) shows the velocity distribution function $P_{\mathbf{v}}(v)$ vs $v / \dot{\gamma}$ with simulation parameters $(\phi, \dot{\gamma})$ and symbols as shown by the inset. The dashed line has slope -3 . Panel (b) shows the part of the dissipated power which is dissipated by the fraction $x$ of the fastest particles. Panel (c) shows the same quantity for the $\mathrm{CD}_{0}$ model. These data are at $\phi=0.8433 \approx$ $\phi_{J}$ and five different shear rates. The insets of panels (b) and (c) are $x_{50}$ - the fraction of particles needed to dissipate $50 \%$ of the power. The inset of panel (b) shows that $x_{50}$ for the $\mathrm{RD}_{0}$ model decreases as $\phi$ increases whereas the inset of panel (c) shows $x_{50}$ at $\phi_{J}$ decreasing with $\dot{\gamma}$ for both $\mathrm{RD}_{0}$ and $\mathrm{CD}_{0}$.

pation due to velocity differences of disks in contact $[3,9]$. Details of these simulations may be found elsewhere[10].

A key quantity in the present letter is the energy dissipation. We here just remark that this is a central quantity due to the relation between dissipation and rheology from power balance, $V \sigma \dot{\gamma}=k_{d}\left\langle\sum_{i} \mathbf{v}_{i}^{2}\right\rangle[11]$, and we therefore believe that the considerations here may be instrumental in developing a better understanding of sheardriven jamming.
Our first key result is that most of the energy is dissipated by a small fraction of fast particles and, furthermore, that the fraction of particles needed to dissipate a given part of the power decreases as jamming is approached. Note that "fast" is here used in a relative sense. For low $\dot{\gamma}$ all particles are slow, it is only $v / \dot{\gamma}$ that can be big. To study the dissipation we introduce the velocity distribution function $P_{\mathbf{v}}(v)$ such that $P_{\mathbf{v}}(v) d v$ is the fraction of particles with velocity $v \leq|\mathbf{v}|<v+d v$. Fig. 1(a) shows $P_{\mathbf{v}}(v)$ vs $v / \dot{\gamma}$ both at five densities below $\phi_{J}$, and for three different shear rates at $\phi_{J}$. (To get histograms of good quality down to small $P_{\mathbf{v}}$ we use bins that are equally spaced in $\ln v$.) The different simulation parameters $(\phi, \dot{\gamma})$ and their corresponding symbols are shown in the inset of panel (a). The points connected by solid lines and dash lines, respectively, show two different ways to approach jamming. The solid line connects $(\phi, \dot{\gamma})$ at $\phi<\phi_{J}$ and at sufficiently low $\dot{\gamma}$ to be very close to the hard disk limit. The dashed line connects three points at $\phi=0.8433 \approx \phi_{J}$. Here jamming is approached as $\dot{\gamma} \rightarrow 0$.

To study the dissipation with focus on the fast particles we define

$$
x(v)=\int_{v}^{\infty} P_{\mathbf{v}}\left(v^{\prime}\right) d v^{\prime}, \quad \bar{C}_{2}(v)=\int_{v}^{\infty} P_{\mathbf{v}}\left(v^{\prime}\right) v^{\prime 2} d v^{\prime} .
$$

Here $x(v)$ is the fraction of fast particles with $|\mathbf{v}|>v$ and $k_{d} \bar{C}_{2}(v)$ is the dissipating power due to the same particles. We also define $C_{2}(x)=\bar{C}_{2}(v(x))$, where $v(x)$ is the inverse of $x(v)$. Fig. 1(b) shows the normalized $C_{2}$ vs $x$ for the data in panel (a). The faster particles always dominate the dissipation but this effect becomes more pronounced - the curves get steeper - as jamming is approached; a smaller fraction of particles is then needed for a given part of the dissipation. As a simple quantitative measure we introduce $x_{50}$, shown in the inset of panel (b), as the fraction of the fastest particles that dissipates $50 \%$ of the power. For the hard disk limit (solid line) $x_{50}$ decreases as $\phi$ increases towards $\phi_{J}$. The behavior of $x_{50}$ at $\phi=0.8433 \approx \phi_{J}$ is shown by the open squares in the inset of panel (c); $x_{50}$ decreases with decreasing $\dot{\gamma}$ and gets as low as $0.16 \%$ at the lowest shear rate, $\dot{\gamma}=10^{-10}$. We believe that this localization of the dissipation to a few faster particles is related to plastic events or avalanches that are found above $\phi_{J}$, as already speculated by others[5].

Panel (c) shows that the $\mathrm{CD}_{0}$ model behaves similarly. In this model it is the velocity differences of contacting particles that is the quantity of interest rather than the non-affine velocity, and $C_{2}$ is defined analogously. The main data in panel (c) is $C_{2}(x)$ at $\phi_{J}$ for the $\mathrm{CD}_{0}$ model which is very similar to the three data sets at $\phi_{J}$ in panel (a). As a more detailed comparison the inset of panel (c) shows $x_{50}$ against $\dot{\gamma}$ at $\phi_{J}$ for both the $\mathrm{RD}_{0}$ model and the $\mathrm{CD}_{0}$ model, and it is clear that this fraction decreases with decreasing $\dot{\gamma}$ in both models. The effect studied here 
is thus not just peculiar to the simpler $\mathrm{RD}_{0}$ model.[12]

The evidence from Fig. 1 strongly suggests that $C_{2}(x) / C_{2}(1)$ approaches a step function as $\phi \rightarrow \phi_{J}$ and $\dot{\gamma} \rightarrow 0$, and this is the main result from the first part of this Letter. For $C_{2}(x) / C_{2}(1)$ to approach a step function the limiting distribution has to have a tail

$$
P_{\mathbf{v}}(v) \sim v^{-3},
$$

since that would make $C_{2}(v)$ diverge. We note that experiments on dense granular flows have led to similar conclusions[13]. Before turning to more elaborate analyses we note that the dashed line in Fig. 1(a) with slope $=-3$ gives some support for Eq. (4) as the limiting behavior at $\phi_{J}$ as $\dot{\gamma} \rightarrow 0$.

For the further analysis it is important to understand the origin of the wide distribution. We note that the nonaffine velocity in the $\mathrm{RD}_{0}$ model is related to the sum of all (repulsive) contact forces that act on the particle. The non-affine velocity of particle $i$ is $\mathbf{v}_{i}=\sum_{j} \mathbf{f}_{i j}^{\mathrm{el}} / k_{d}$. Close to jamming, the forces on most particles almost cancel one another out, and the total force is typically very small compared to the average force, $f_{i}^{\mathrm{el}} \ll f_{i j}^{\mathrm{el}}$, as has also been noted by others[14]. There are however some particles for which the forces don't balance one another out, and the velocity of these particles can then be much larger than the average velocity. The wide distribution is thus due to the big difference between the individual forces and the typical total force.

A consequence of this picture is that the maximum velocity is bounded by the typical $f_{i j}^{\text {el }}$ which means that the possibly algebraic distribution is cut off by an exponential factor $e^{-v / v_{c}}$, where $v_{c} \sim f_{i j} / k_{d} \sim p /\left(k_{d} d_{s}\right)$. (This also suggests $v_{c} / \dot{\gamma} \sim \eta_{p}$.) This behavior is seen in Fig. 1(a) as the approximately rectilinear (i.e. algebraic) behaviors for intermediate values of $P_{\mathbf{v}}(v)$ turn into more rapid decays at higher velocities. One therefore expects the tails in the distributions to be described by $P(v) \sim v^{-r} e^{-v / v_{c}}$, and this exponential decay becomes a complicating factor, when one attempts to determine $r$ from $P(v)$.

Our second key result is that different measures of the velocity behave differently. This is important since it means that concepts like "typical velocity" —used in various theoretical approaches - then become useless. Fig. 2 shows simulation results for $\tilde{v}_{1}$ and $\tilde{v}_{\text {rms }}$, defined through

$$
\tilde{v}_{1}=\langle|\tilde{\mathbf{v}}|\rangle, \quad \text { and } \quad \tilde{v}_{\mathrm{rms}}^{2}=\left\langle\tilde{\mathbf{v}}^{2}\right\rangle \text {, }
$$

with the notation $\tilde{\mathbf{v}}=\mathbf{v} / \dot{\gamma}$. In panel (a) these quantities are plotted against $\dot{\gamma}$ and are found to diverge algebraically with different exponents: $\tilde{v}_{\text {rms }} \sim \dot{\gamma}^{-\beta / 2 z \nu} \sim$ $\dot{\gamma}^{-0.34}$ and $\tilde{v}_{1} \sim \dot{\gamma}^{-u_{v} / z \nu} \sim \dot{\gamma}^{-0.28}$. [The expressions follow by taking $b=\dot{\gamma}^{-1 / z}$ in $\mathcal{O}(\phi, \dot{\gamma})=b^{u_{\mathcal{O}} / \nu} g_{\mathcal{O}}\left(\left(\phi_{J}-\right.\right.$ $\left.\phi) b^{1 / \nu}, \dot{\gamma} b^{z}\right)[15]$ with the scaling dimension $u_{\mathcal{O}}$ equal to $u_{v}$ for $\tilde{v}_{1}$ and $\beta / 2$ for $\tilde{v}_{\text {rms. }}$. The latter follows from $\eta \sim \tilde{v}_{\text {rms }}^{2}$ and $\eta(\phi, \dot{\gamma} \rightarrow 0) \sim\left(\phi_{J}-\phi\right)^{-\beta}$.]

It is instructive to also examine the same quantities with data below $\phi_{J}$, close to the hard disk limit. The
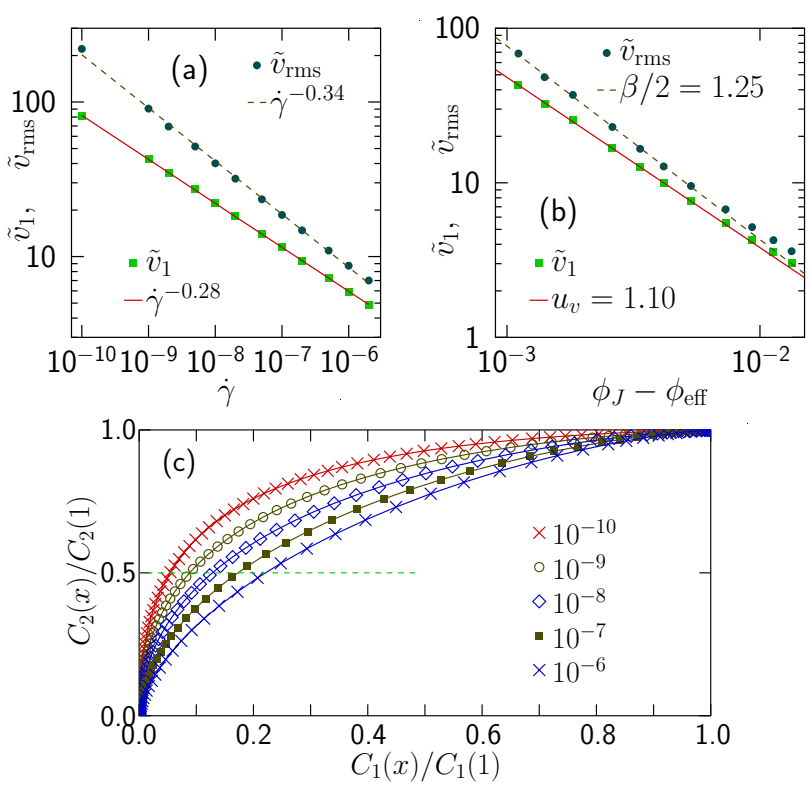

FIG. 2. (Color online) Two different measures of the velocity. Panel (a) shows $\tilde{v}_{\text {rms }}$ and $\tilde{v}_{1}$ for $\phi=0.8433 \approx \phi_{J}$ vs $\dot{\gamma}$. Panel (b) shows the same quantities for data below $\phi_{J}$ plotted vs distance to jamming; only the points with $\phi_{J}-\phi_{\text {eff }}<0.006$ were used for the fits. $\phi_{\text {eff }}$ is the effective density. The lines are $\sim\left(\phi_{J}-\phi_{\text {eff }}\right)^{-u_{v}}$ and $\sim\left(\phi_{J}-\phi_{\text {eff }}\right)^{-\beta / 2}$. Panel (c) connects back to Fig. 1(a) but is $C_{2}$, related to $\tilde{v}_{\text {rms }}^{2}$, against $C_{1}$ which is related to $\tilde{v}_{1}$. From the crossings of the dashed line one may read off the relative contribution to $\tilde{v}_{1}$ from the fraction of the fastest particles that disspate $50 \%$ of the energy - a quantity that decreases with decreasing $\dot{\gamma}$.

starting point is the relations for hard disks, $\tilde{v}_{1}^{\text {hd }}(\phi) \sim$ $\left(\phi_{J}-\phi\right)^{-u_{v}}$, and $\tilde{v}_{\mathrm{rms}}^{\mathrm{hd}}(\phi) \sim\left(\phi_{J}-\phi\right)^{-\beta / 2}$, that follow by using $b=\dot{\gamma}$ in the scaling expressions and considering $\dot{\gamma} \rightarrow 0$. In Fig. 2(b) we make use of the effective-density mapping of soft disks onto hard disks, $\mathcal{O}^{\text {hd }}\left(\phi_{\text {eff }}\right)=$ $\mathcal{O}(\phi, \dot{\gamma})$, where the effective density is $\phi_{\text {eff }}=\phi-c E^{1 / 2 y}$, with $c=1.53$ and $y=1.09$, as detailed in Ref. [16]. Fig. 2(b) shows $\tilde{v}_{1}$ and $\tilde{v}_{\text {rms }}$ against $\phi_{J}-\phi_{\text {eff. }}$ The solid line gives the exponent $u_{v}=1.10$ in agreement with $\ell_{\Delta} \sim\left(\phi_{J}-\phi\right)^{-1.1}$ for the particle "velocity" in Ref. [5]. The dashed line gives $\beta / 2=1.25$. (The value $\beta=2.50$, is somewhat low in comparison to recent estimates[15], but this could be due to not including corrections to scaling[15].) Note that the exponents from Fig. 2(a) and (b) are consistent when using $1 / z \nu=0.26[15]$.

The reason for the different behavior of $\tilde{v}_{1}$ and $\tilde{v}_{\text {rms }}$ is that the dominant contribution to these quantities come from different velocity intervals. This is illustrated in Fig. 2(c) which shows how $C_{2}(x)$ in Eq. (3) and $C_{1}(x)$ (for $v^{1}$ instead of $v^{2}$ ) increase to their respective limits $C_{2}(1) \equiv \tilde{v}_{\text {rms }}^{2}$ and $C_{1}(1) \equiv \tilde{v}_{1}$, as $x$ (the fraction of particles included in the calculations) increases. The different curves get steeper for smaller $\dot{\gamma}$ and for $\dot{\gamma}=10^{-10}$ we find $C_{1}(x) \approx 0.05$ when $C_{2}(x)=0.5$ which thus shows that $\tilde{v}_{\text {rms }}^{2}$ gets a considerably larger contribution from the 

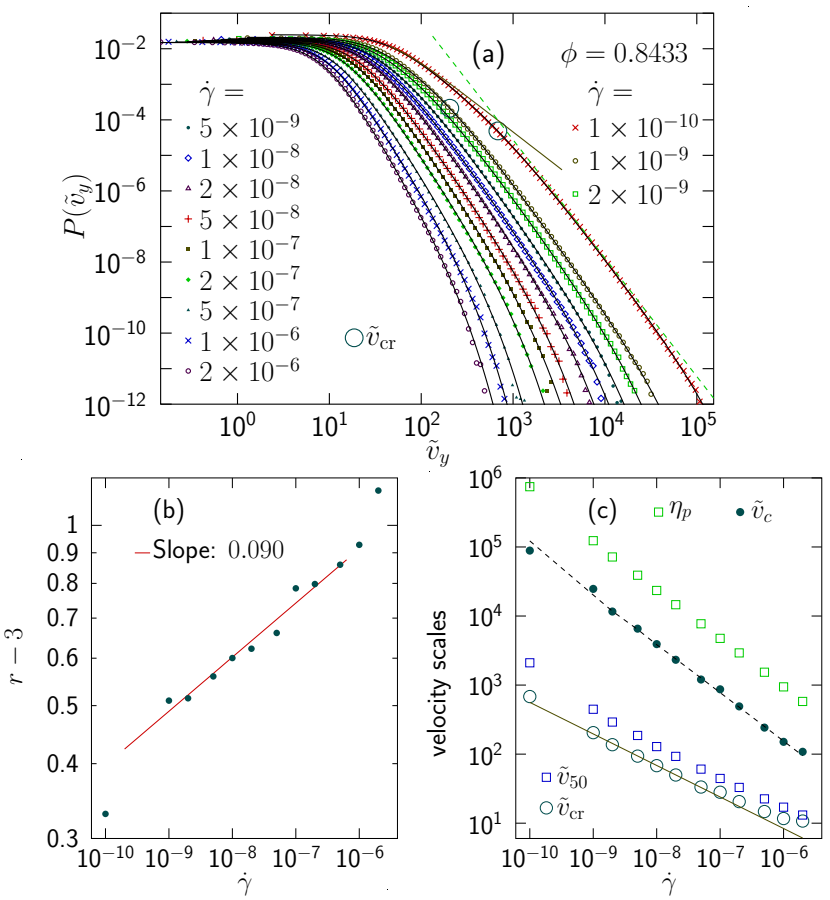

FIG. 3. (Color online) Velocity distribution and the determination of the exponent $r$ for different $\dot{\gamma}$ at $\phi=0.8433 \approx \phi_{J}$. Panel (a) is the velocity distribution $P\left(\tilde{v}_{y}\right)$. Panel (b) shows $\tilde{v}_{c}$ and $r-3$ vs $\dot{\gamma}$ from fitting to Eq. (6). Fitting $r-3 \sim \dot{\gamma}^{q_{r}}$ for $10^{-9} \leq \dot{\gamma} \leq 5 \times 10^{-7}$ gives $q_{r}=0.09 \pm 0.02$. Panel (c) shows that $\eta_{p}$ and $\tilde{v}_{c}$ are proportional - the dashed line through $\tilde{v}_{c}$ is $\eta_{p} / 6.1$. Also shown are $\tilde{v}_{\mathrm{cr}}-$ which is where the histogram crosses over to exponent $-r$, and $\tilde{v}_{50}$ above which $50 \%$ of the dissipation takes place. These similarity of these two quantities suggests that the dominant part of the dissipation is given by particles governed by the algebraic tail.

highest velocity part of the histogram than $\tilde{v}_{1}$. An extrapolation of these curves to the $\dot{\gamma} \rightarrow 0$ limit would give a step function (though this is not as clearly suggested by the data as in Fig. 1) which would imply that $\tilde{v}_{\text {rms }}^{2}$ and $\tilde{v}_{1}$ were controlled by different velocity intervals, and that there is no reason for these quantities to be at all related.

We will now relate our two key results of Figs. 1 and 2 to properties of the velocity distribution function with the goal (1) to examine how the exponent in Eq. (4) approaches -3 as $\dot{\gamma} \rightarrow 0$ (this exponent will be denoted by $-r)$ and (2) to shed some more light on the mechanism that allows $\tilde{v}_{1}$ and $\tilde{v}_{\text {rms }}$ to diverge differently. We have then found it convenient to use $P\left(\tilde{v}_{y}\right)$ - the distribution of the absolute value of the $y$-component. This quantity differs from $P_{\mathbf{v}}$ in that it approaches a constant at small velocities - a feature that makes it easier to find an analytical expression that fits the data. Fig. 3(a) shows $P\left(\tilde{v}_{y}\right)$ for several different $\dot{\gamma}$ at $\phi=0.8433 \approx \phi_{J}$ together with the solid lines that are fits to the expression

$$
P\left(\tilde{v}_{y}\right)=\frac{A e^{-\tilde{v}_{y} / \tilde{v}_{c}}}{1+\left(\tilde{v}_{y} / \tilde{v}_{a}\right)^{2}+\left(\tilde{v}_{y} / \tilde{v}_{s}\right)^{r}},
$$

with $A, \tilde{v}_{c}, \tilde{v}_{a}, \tilde{v}_{s}$, and $r$ as free parameters. This expression crosses over from a constant at small $\tilde{v}_{y}$ to a large- $\tilde{v}_{y}$ tail with $\tilde{v}_{y}^{-r} e^{-\tilde{v}_{y} / \tilde{v}_{c}}$ (as discussed above) and the crossover is governed by an additional term in the denominator, $\left(\tilde{v}_{y} / \tilde{v}_{a}\right)^{a}$. For best possible fits, $a$ should be an additional free parameter, but since $a$ anyway tends to be close to 2 and $a=2$ opens up for analytical calculations[17], we here fix $a=2$.

Fig. 3(b) shows the exponent as $r-3$ vs $\dot{\gamma}$. The rectilinear behavior suggests an algebraic decay, $r-3 \sim \dot{\gamma}^{q_{r}}$ with $q_{r}=0.09 \pm 0.02$, consistent with the limiting behavior of Eq. (4). The points on top of Fig. 3(c) are the cutoff velocity, $\tilde{v}_{c}$ (solid circles) from the fits and $\eta_{p}$ (squares) directly from simulations. The dashed line through the solid circles, which is $\eta_{p} / 6.1$ and not a fit to the data, confirms the expectation that these quantities should behave the same. The covariation of $r$ and $\tilde{v}_{c}$ (compare panels (b) and (c)) makes the fitting difficult - a small decrease in $r$ can be compensated by a small decrease in $\tilde{v}_{c}$ since a smaller $r$ gives a slower decay, while a smaller $\tilde{v}_{c}$ gives a faster decay. This effect is most problematic at the lowest shear rate, $\dot{\gamma}=10^{-10}$, and this point is therefore not included in the determination of $q_{r}$.

It is now interesting to determine the size of the region governed by the algebraic decay, $\tilde{v}_{y}^{-r}$, and we therefore calculate the crossover velocity $\tilde{v}_{\mathrm{cr}}=\left(\tilde{v}_{s}^{r} / \tilde{v}_{a}^{2}\right)^{1 /(r-2)}$, that describes the crossover from exponent -2 to exponent $-r$, by equating the two velocity-dependent terms in the denominator of Eq. (6). Fig. 3(c) shows that $\tilde{v}_{\mathrm{cr}}$ behaves about the same as $\tilde{v}_{50}$-related to $x_{50}$ abovewhich is the velocity above which $50 \%$ of the dissipation takes place. We thus find that the dissipation is largely governed by the particles in the algebraic tail. Recalling the conclusions from Fig. 1, it is clear that the fraction of particles in the algebraic tail decreases with $\dot{\gamma}$ and this is also shown by the big open circles in Fig. 3(a) which are $P\left(\tilde{v}_{\text {cr }}\right)$ vs $\tilde{v}_{\text {cr }}$ for $\dot{\gamma}=10^{-9}$ and $10^{-10}$ — the last two points in a persistent trend to smaller $P\left(\tilde{v}_{\text {cr }}\right)$. The fact that the fraction of particles in the algebraic tail decreases with decreasing $\dot{\gamma}$ means that their contribution to $\tilde{v}_{1}$ decreases ( $\tilde{v}_{1}$ is instead dominated by the slower particles) whereas they always give the dominant contribution to $\tilde{v}_{\mathrm{rms}}^{2}$ [17]. This explains the different behavior of $\tilde{v}_{1}$ and $\tilde{v}_{\text {rms. }}$. We finally note that the algebraic tail actually becomes wider as $\dot{\gamma}$ decreases. Fig. 3(c) shows that $\tilde{v}_{c}$ increases faster than $\tilde{v}_{\text {cr }}$ which means that the width of the algebraic tail - the region between $\tilde{v}_{\text {cr }}$ and $\tilde{v}_{c}$ - increases with decreasing $\dot{\gamma}$.

To summarize, we have found that the fraction of particles that are responsible for the energy dissipation decreases towards zero as jamming is approached. These particles belong to a tail in the velocity distribution that approaches $P(v) \sim v^{-3}$ at jamming. We further find that different measures of the velocity diverge differently which means that concepts like "typical velocity" no longer appear to be useful - a result of importance for 
analytical approaches to shear-driven jamming.

I thank S. Teitel for many illuminating discussions. This work was supported by the Swedish Research Council Grant No. 2010-3725. Simulations were performed on resources provided by the Swedish National Infrastructure for Computing (SNIC) at PDC and HPC2N.

[1] A. J. Liu and S. R. Nagel, Nature (London) 396, 21 (1998)

[2] C. S. O'Hern, L. E. Silbert, A. J. Liu, and S. R. Nagel, Phys. Rev. E 68, 011306 (2003)

[3] D. J. Durian, Phys. Rev. Lett. 75, 4780 (1995)

[4] E. Lerner, G. Düring, and M. Wyart, PNAS 109, 4798 (2012)

[5] C. Heussinger, L. Berthier, and J.-L. Barrat, Europhys. Lett. 90, 20005 (2010)

[6] B. Andreotti, J.-L. Barrat, and C. Heussinger, Phys. Rev. Lett. 109, 105901 (2012)

[7] G. Marty and O. Dauchot, Phys. Rev. Lett. 94, 015701
(2005)

[8] D. J. Evans and G. P. Morriss, Statistical Mechanics of Nonequilibrium Liquids (Academic Press, London, 1990)

[9] B. P. Tighe, E. Woldhuis, J. J. C. Remmers, W. van Saarloos, and M. van Hecke, Phys. Rev. Lett. 105, 088303 (2010)

[10] D. Vågberg, P. Olsson, and S. Teitel, Phys. Rev. Lett. 113, 148002 (2014)

[11] I. K. Ono, S. Tewari, S. A. Langer, and A. J. Liu, Phys. Rev. E 67, 061503 (2003)

[12] Preliminary analyses of data from three-dimensional systems suggest that they behave qualitatively the same. D. Vågberg and P. Olsson, unpublished.

[13] S. Moka and P. R. Nott, Phys. Rev. Lett. 95, 068003 (2005)

[14] E. DeGiuli, G. Düring, E. Lerner, and M. Wyart, Phys. Rev. E 91, 062206 (2015)

[15] P. Olsson and S. Teitel, Phys. Rev. E 83, 030302(R) (2011)

[16] P. Olsson and S. Teitel, Phys. Rev. Lett. 109, 108001 (2012)

[17] See the Supplemental material for further details. 


\section{Supplemental material}

Derivations related to "Dissipation and velocity distribution at the shear-driven jamming transition", Peter Olsson.

\section{Summary}

The purpose of the calculations below is to illustrate the mechanism that gives different behaviors for $\tilde{v}_{1}$ and $\tilde{v}_{\text {rms }}$. The idea is here to split the expression for the velocity distribution

$$
P\left(\tilde{v}_{y}\right)=\frac{e^{-\tilde{v}_{y} / \tilde{v}_{c}}}{1+\left(\tilde{v}_{y} / \tilde{v}_{a}\right)^{2}+\left(\tilde{v}_{y} / \tilde{v}_{s}\right)^{r}},
$$

into three different parts

$$
P\left(\tilde{v}_{y}\right)= \begin{cases}1 /\left[1+\left(\tilde{v}_{y} / \tilde{v}_{a}\right)^{2}\right], & 0 \leq \tilde{v}_{y}<\tilde{v}_{\mathrm{cr}} \\ 1 /\left(\tilde{v}_{y} / \tilde{v}_{s}\right)^{r}, & \tilde{v}_{\mathrm{cr}}<\tilde{v}_{y}<\tilde{v}_{c} \\ 0, & \tilde{v}_{c}<\tilde{v}_{y} .\end{cases}
$$

We may then determine $\tilde{v}_{1}=\left\langle\tilde{v}_{y}\right\rangle$ and $\tilde{v}_{\text {rms }}^{2}=\left\langle\tilde{v}_{y}^{2}\right\rangle$ by calculating some integrals analytically, as shown below. The results are then written in terms of the different velocity scales, $\tilde{v}_{a}, \tilde{v}_{s}, \tilde{v}_{\mathrm{cr}}$, and $\tilde{v}_{c}$. Fig. 4 and Fig. 3(c) show that all these quantities to decent approximations diverge algebraically for small $\dot{\gamma}$. We have $\tilde{v}_{a} \sim \dot{\gamma}^{-q_{a}}, \tilde{v}_{s} \sim \dot{\gamma}^{-q_{s}}, \tilde{v}_{\text {cr }} \sim \dot{\gamma}^{-q_{\mathrm{cr}}}$, and $\tilde{v}_{c} \sim \dot{\gamma}^{-q_{c}}$ with the exponents $q_{a} \approx 0.18, q_{s} \approx 0.29, q_{\mathrm{cr}} \approx 0.46$, and $q_{c} \approx 0.71$.

The first moment of the velocity becomes

$$
\tilde{v}_{1}=\left\langle\tilde{v}_{y}\right\rangle \approx \tilde{v}_{a}\left[\ln \left(\frac{\tilde{v}_{\mathrm{cr}}}{\tilde{v}_{a}}\right)+\frac{1}{r-2}\right] \approx \tilde{v}_{a}\left[0.62 \log _{10}(1 / \dot{\gamma})+\frac{1}{r-2}\right] .
$$

For the second moment of the velocity we get different results when $r$ is close to 3. For $r$ not too close to 3 (which is the case relevant for comparisions with our simulation data) we find

$$
\tilde{v}_{\mathrm{rms}}^{2} \approx \tilde{v}_{a} \tilde{v}_{\mathrm{cr}}\left[1+\frac{1}{r-3}\right]
$$

whereas for $r \rightarrow 3$ we instead find

$$
\begin{aligned}
\tilde{v}_{\mathrm{rms}}^{2} & \approx \tilde{v}_{a} \tilde{v}_{\mathrm{cr}}+\frac{\tilde{v}_{s}^{3}}{\tilde{v}_{a}} \ln \left(\frac{\tilde{v}_{c}}{\tilde{v}_{\mathrm{cr}}}\right) \approx \tilde{v}_{a} \tilde{v}_{\mathrm{cr}}\left[1+\left(q_{c}-q_{\mathrm{cr}}\right) \ln (1 / \dot{\gamma})\right] \\
& \approx \tilde{v}_{a} \tilde{v}_{\mathrm{cr}}\left[1+0.58 \log _{10}(1 / \dot{\gamma})\right] .
\end{aligned}
$$




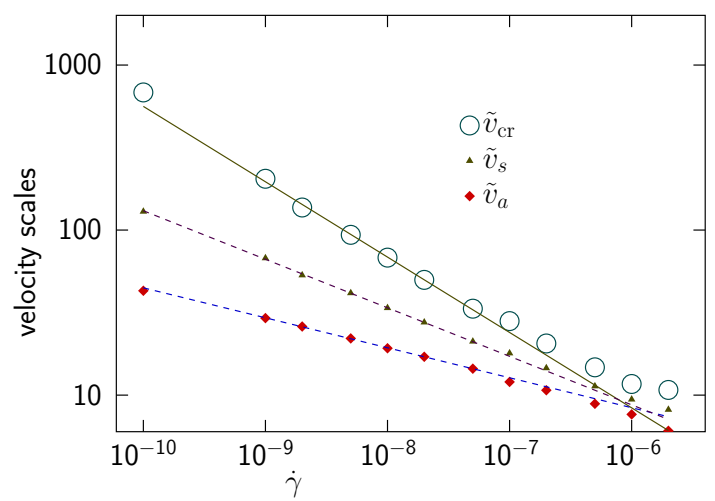

Figure 4: Velocity scales $\tilde{v}_{a}, \tilde{v}_{s}$, and $\tilde{v}_{\text {cr }}$ of Eq. (6). All these quantities behave algebraically for the smaller $\dot{\gamma}$, to decent approximations.

\section{The small- $\dot{\gamma}$ limit}

With the values for the exponents given above, the leading small- $\dot{\gamma}$ behaviors become $\tilde{v}_{\text {rms }}^{2} \sim \tilde{v}_{a} \tilde{v}_{\text {cr }} \sim \dot{\gamma}^{-\left(q_{\mathrm{cr}}+q_{a}\right) / 2} \sim \dot{\gamma}^{-0.64}$ in very good agreement with $\tilde{v}_{\text {rms }} \sim \dot{\gamma}^{-0.34}$ in Fig. 2(a).

For $\tilde{v}_{1}$, the full expression $\tilde{v}_{1} \sim \tilde{v}_{a}\left[\ln \left(\tilde{v}_{\text {cr }} / \tilde{v}_{a}\right)+1 /(r-2)\right]$ reproduces the exponent -0.28 in Fig. 2(a). In the $\dot{\gamma} \rightarrow 0$ limit, however, the second term vanishes and the slowly changing logarithm may be replaced by a constant, and this gives $\tilde{v}_{1} \sim \dot{\gamma}^{-q_{a}} \sim \dot{\gamma}^{-0.18}$, which is clearly different from the measured exponent, -0.28 . We consider the exponent obtained directly from the measured data to be more reliable as it is from an excellent fit to data across four orders of magnitude in $\dot{\gamma}$. In contrast, the "analytical" value for the exponent $(-0.18)$ is obtained by the dangerous process of extrapolating results from fitting to an expression (Eq. (6)) that is used without any theoretical justification. 


\section{Derivations}

To determine $\tilde{v}_{1} \equiv\left\langle\tilde{v}_{y}\right\rangle$ and $\tilde{v}_{\text {rms }}^{2}=\left\langle\tilde{v}_{y}^{2}\right\rangle$ we need the integrals

$$
I_{0}=\int P\left(\tilde{v}_{y}\right) d \tilde{v}_{y}, \quad I_{1}=\int P\left(\tilde{v}_{y}\right) \tilde{v}_{y} d \tilde{v}_{y}, \quad I_{2}=\int P\left(\tilde{v}_{y}\right) \tilde{v}_{y}^{2} d \tilde{v}_{y},
$$

which with Eq. (7) becomes

$$
I_{p}=\int P\left(\tilde{v}_{y}\right) \tilde{v}_{y}^{p} d \tilde{v}_{y}=\int_{0}^{\tilde{v}_{\mathrm{cr}}} \frac{\tilde{v}_{y}^{p}}{1+\left(\tilde{v}_{y} / \tilde{v}_{a}\right)^{2}} d \tilde{v}_{y}+\int_{\tilde{v}_{\mathrm{cr}}}^{\tilde{v}_{c}} \tilde{v}_{y}^{p}\left(\tilde{v}_{y} / \tilde{v}_{s}\right)^{-r} d \tilde{v}_{y} .
$$

It is then convenient to consider the two terms above separately.

First term, $I_{p}^{(1)}$

We here use $x=\tilde{v}_{y} / \tilde{v}_{a}, d x=d \tilde{v}_{y} / \tilde{v}_{a}$, and $x_{\mathrm{cr}}=\tilde{v}_{\mathrm{cr}} / \tilde{v}_{a}$ and handle the different integrals separately for different $p$ :

- With $p=0$ :

$$
\begin{aligned}
I_{0}^{(1)}=\int_{0}^{\tilde{v}_{\mathrm{cr}}} \frac{1}{1+\left(\tilde{v}_{y} / \tilde{v}_{a}\right)^{2}} d \tilde{v}_{y} & =\tilde{v}_{a} \int_{0}^{x_{\mathrm{cr}}} \frac{1}{1+x^{2}} d x=\tilde{v}_{a}[\arctan x]_{0}^{x_{\mathrm{cr}}} \\
& =\tilde{v}_{a} \arctan \left(\tilde{v}_{\mathrm{cr}} / \tilde{v}_{a}\right)
\end{aligned}
$$

- With $p=1$ :

$$
\begin{aligned}
I_{1}^{(1)}=\int_{0}^{\tilde{v}_{\mathrm{cr}}} \frac{\tilde{v}_{y}}{1+\left(\tilde{v}_{y} / \tilde{v}_{a}\right)^{2}} d \tilde{v}_{y} & =\tilde{v}_{a}^{2} \int_{0}^{x_{\mathrm{cr}}} \frac{x}{1+x^{2}} d x=\tilde{v}_{a}^{2}\left[\frac{1}{2} \ln \left(1+x^{2}\right)\right]_{0}^{x_{\mathrm{cr}}} \\
& =\frac{\tilde{v}_{a}^{2}}{2} \ln \left(1+\left(\tilde{v}_{\mathrm{cr}} / \tilde{v}_{a}\right)^{2}\right) .
\end{aligned}
$$

- With $p=2$ :

$$
\begin{aligned}
I_{2}^{(1)}=\int_{0}^{\tilde{v}_{\mathrm{cr}}} \frac{\tilde{v}_{y}^{2}}{1+\left(\tilde{v}_{y} / \tilde{v}_{a}\right)^{2}} d \tilde{v}_{y} & =\tilde{v}_{a}^{3} \int_{0}^{x_{\mathrm{cr}}} \frac{x^{2}}{1+x^{2}} d x=\tilde{v}_{a}^{3}[x-\arctan x]_{0}^{x_{\mathrm{cr}}} \\
& =\tilde{v}_{a}^{2} \tilde{v}_{\mathrm{cr}}-\tilde{v}_{a}^{3} \arctan \left(\tilde{v}_{\mathrm{cr}} / \tilde{v}_{a}\right) .
\end{aligned}
$$


Second term, $I_{p}^{(2)}$

We here get an expression for general $p$ :

$$
\begin{aligned}
I_{p}^{(2)}=\int_{\tilde{v}_{\mathrm{cr}}}^{\tilde{v}_{c}} \tilde{v}_{y}^{p}\left(\tilde{v}_{y} / \tilde{v}_{s}\right)^{-r} d \tilde{v}_{y} & =\tilde{v}_{s}^{r}\left[\frac{\tilde{v}_{y}^{p+1-r}}{p+1-r}\right]_{\tilde{v}_{\mathrm{cr}}}^{\tilde{v}_{c}} \\
& =\frac{\tilde{v}_{s}^{r}}{p+1-r}\left(\tilde{v}_{c}^{p+1-r}-\tilde{v}_{\mathrm{cr}}^{p+1-r}\right) \\
& =\frac{\tilde{v}_{s}^{p+1}}{r-1-p}\left[\left(\frac{\tilde{v}_{s}}{\tilde{v}_{\mathrm{cr}}}\right)^{r-1-p}-\left(\frac{\tilde{v}_{s}}{\tilde{v}_{c}}\right)^{r-1-p}\right] .
\end{aligned}
$$

This splits into two cases. If $r-1-p \gg 0$ (which is always the case in our simulations) and $\tilde{v}_{c} \gg \tilde{v}_{\text {cr }}$ (which allows us to skip the second term) we make use of $\tilde{v}_{\mathrm{cr}}^{r-2}=\tilde{v}_{s}^{r} / \tilde{v}_{a}^{2}$ to get

$$
I_{p}^{(2)} \approx \frac{\tilde{v}_{s}^{p+1}}{r-1-p}\left(\frac{\tilde{v}_{s}}{\tilde{v}_{\mathrm{cr}}}\right)^{r-1-p}=\frac{\tilde{v}_{a}^{2} \tilde{v}_{\mathrm{cr}}^{p-1}}{r-1-p} .
$$

For $r-1-p \approx 0$, on the other hand, (possible for $p=2$ and very close to jamming, $r \rightarrow 3$ ), we get

$$
I_{p}^{(2)} \approx \tilde{v}_{s}^{p+1}\left[\ln \left(\frac{\tilde{v}_{s}}{\tilde{v}_{\mathrm{cr}}}\right)-\ln \left(\frac{\tilde{v}_{s}}{\tilde{v}_{c}}\right)\right]=\tilde{v}_{s}^{p+1} \ln \left(\frac{\tilde{v}_{c}}{\tilde{v}_{\mathrm{cr}}}\right)
$$

\section{Taking both terms together}

The normalization becomes

$$
I_{0}=\tilde{v}_{a} \arctan \left(\frac{\tilde{v}_{\mathrm{cr}}}{\tilde{v}_{a}}\right)+\frac{1}{r-1} \frac{\tilde{v}_{a}^{2}}{\tilde{v}_{\mathrm{cr}}} \approx \frac{\pi}{2} \tilde{v}_{a}
$$

For the first moment we get

$$
\begin{aligned}
I_{1} & =\frac{\tilde{v}_{a}^{2}}{2} \ln \left[1+\left(\frac{\tilde{v}_{\mathrm{cr}}}{\tilde{v}_{a}}\right)^{2}\right]+\frac{\tilde{v}_{a}^{2}}{r-2} \\
& \approx \tilde{v}_{a}^{2}\left[\ln \left(\frac{\tilde{v}_{\mathrm{cr}}}{\tilde{v}_{a}}\right)+\frac{1}{r-2}\right] .
\end{aligned}
$$

The logarithmic correction is $\ln \left(\tilde{v}_{\mathrm{cr}} / \tilde{v}_{a}\right)=\ln \left(\dot{\gamma}^{-q_{\mathrm{cr}}} / \dot{\gamma}^{-q_{a}}\right) \sim\left(q_{\mathrm{cr}}-q_{a}\right) \ln (1 / \dot{\gamma})$. The average velocity becomes (using $q_{a} \approx 0.18$ and $q_{\mathrm{cr}} \approx 0.46$ )

$$
\tilde{v}_{1}=\left\langle\tilde{v}_{y}\right\rangle=I_{1} / I_{0} \approx \tilde{v}_{a}\left[\ln \left(\frac{\tilde{v}_{\mathrm{cr}}}{\tilde{v}_{a}}\right)+\frac{1}{r-2}\right] \approx \tilde{v}_{a}\left[0.62 \log _{10}(1 / \dot{\gamma})+\frac{1}{r-2}\right] .
$$


For the second moment and for $r$ not too close to 3, Eq. (13) leads to

$$
I_{2}=\tilde{v}_{a}^{2} \tilde{v}_{\mathrm{cr}}\left[1+\frac{1}{r-3}\right]-\tilde{v}_{a}^{3} \arctan \left(\frac{\tilde{v}_{\mathrm{cr}}}{\tilde{v}_{a}}\right),
$$

and since $\arctan x<\pi / 2$, the second term may be neglected and we get

$$
\left\langle\tilde{v}_{y}^{2}\right\rangle \equiv \tilde{v}_{\mathrm{rms}}^{2} \approx \tilde{v}_{a} \tilde{v}_{\mathrm{cr}}\left[1+\frac{1}{r-3}\right],
$$

For $r \rightarrow 3$ we instead use Eq. (14) which gives (with $\tilde{v}_{\text {cr }} \approx \tilde{v}_{s}^{3} / \tilde{v}_{a}^{2}$ and $q_{c}=$ $0.71)$.

$$
\begin{aligned}
\left\langle\tilde{v}_{y}^{2}\right\rangle \equiv \tilde{v}_{\mathrm{rms}}^{2} & \approx \tilde{v}_{a} \tilde{v}_{\mathrm{cr}}+\frac{\tilde{v}_{s}^{3}}{\tilde{v}_{a}} \ln \left(\frac{\tilde{v}_{c}}{\tilde{v}_{\mathrm{cr}}}\right) \approx \tilde{v}_{a} \tilde{v}_{\mathrm{cr}}\left[1+\left(q_{c}-q_{\mathrm{cr}}\right) \ln (1 / \dot{\gamma})\right] \\
& \approx \tilde{v}_{a} \tilde{v}_{\mathrm{cr}}\left[1+0.58 \log _{10}(1 / \dot{\gamma})\right] .
\end{aligned}
$$

